\title{
ANALYSIS OF REACTIVE AGILITY AND CHANGE-OF-DIRECTION SPEED BETWEEN SOCCER PLAYERS AND PHYSICAL EDUCATION STUDENTS
}

original paper

( ) University School of Physical Education in Wroclaw

DOI: https://doi.org/10.5114/hm.2018.74061

\section{SAMO RAUTER ${ }^{1}$, MILAN COH ${ }^{1}$, JANEZ VODICAR ${ }^{1}$, MILAN ZVAN ${ }^{1}$, JOSEF KRIZAJ ${ }^{1}$,} JOZEF SIMENKO ${ }^{1}$, LUKASZ SZMAJDA ${ }^{2}$, KRZYSZTOF MACKALA ${ }^{2}$

${ }^{1}$ Faculty of Sport, University of Ljubljana, Ljubljana, Slovenia

${ }^{2}$ Department of Track and Field, University School of Physical Education, Wroclaw, Poland

\section{ABSTRACT}

Purpose. The main goal of the experiment was to examine the differences between reactive agility (RA) as non-planned (randomly selected stimuli) movement and change-of-direction speed (CODS) as pre-planned movement among different levels of sports performance - young soccer players and physical education students.

Methods. The two groups in the study included 36 young male and female soccer players and 58 male and female physical education students. Timing for universal agility was defined by two different specification: pre-planned agility as a change of direction speed and non-planned (randomly selected stimuli) agility as reactive agility. The study used FitLight Trainer, a wireless system of interconnected light powered sensors.

Results. Soccer players were significantly faster in selected test of CODS (-1.73s; 13.1\%) and RA (-2.34s; $14.3 \%)$. The greatest difference between male and female subjects was observed in the non-planned universal reactive agility UA-RA $(-2.84 \mathrm{~s} ; 17.9 \%)$. A t-test indicated statistically significant gender differences in the selected test of pre-planned (non-reactive) and non-planned (reactive) agility among soccer players and students.

Conclusions. The practical message for coaches and related professionals is that agility in team sports (including soccer) as motor skills represent various physical qualities. Therefore, it should be diagnosed and developed via separate assessments and training.

Key words: reactive agility, non-reactive agility, CODS, FitLight

\section{Introduction}

Soccer represents one of the most popular team sports in the world with an increasing numbers of youth players that play soccer in organized programs $[1,2]$. Soccer performance is a complex interplay of multiple interacting skills and qualities [3]. The intermittent nature of soccer places a major demand on the physiological requirements of both the cardiovascular and metabolic capacities of soccer players [4]. Most playtime in soccer is devoted to aerobic activities, but anaerobic metabolism also plays an important part in the relevant actions of soccer, such as sprint- ing with and without the ball or changing direction [5]. Soccer requires athletes to perform short sprints, repeatedly change directions, and complete numerous jumps during a 90 minute match. For example, several investigators have demonstrated that selected players perform better on linear sprint, agility or jump tests compared with sub-elite and non-selected players, indicating the importance of these performance characteristics for soccer [6-8].

Agility is recognized as a very complex biomotor ability, which consists of several components and is mainly dependent on internal and external factors. Agility is an essential element of many sports disci-

Correspondence address: Krzysztof Mackala, Department of Track and Field, University School of Physical Education, Wroclaw, Poland, e-mail: krzysztof.mackala@awf.wroc.pl

Received: November 16, 2017

Accepted for publication: January 24, 2018

Citation: Rauter S, Coh M, Vodicar J, Zvan M, Krizaj J, Simenko J, Szmajda L, Mackala K. Analysis of reactive agility and change-of-direction speed between soccer players and physical education students. Hum Mov. 2018;19(2):68-74; doi: https://doi.org/10.5114/hm.2018.74061. 
plines that are composed of complementary movement structures [9]. In particular, agility can be defined as the ability to change the direction of the body rapidly and is a result of a combination of strength, speed, balance and coordination. Numerous studies [9-11] indicate that performance where change of direction movements play a significant role does not depend solely on physical abilities and special skills, but cognitive factors are also of great importance. Many authors [12-14] claim that the cognitive look at the problem of agility is very close related with visual perceptual speed, sensory memory kinaesthetic recognition of the space, selective focus, as well as anticipation. Therefore, athletes who demonstrate exceptional motor abilities and special skills should be able to perform high quality agility movements based on their ability to integrate these components via cognitive functioning $[10,15,16]$. Agility performance is of great relevance in soccer given its multidirectional nature that is mostly overlooked in applied research [1]. Agility tests play a key role as indicators of performance in soccer and tend to provide a differentiation between non-players, recreational, and elite players [17, 18]. Moreover, agility performance has been noted to be a predictor of playing level in youth soccer, including differentiation of athletes that drop out of the sport $[19,20]$.

Sport-specific tasks executed during the game are generally complex with a very high dynamics coefficient of execution and a certain level of risk. In most cases, they are performed under high stress, in a not fully recognized environment and only allow for limited time to take concrete action. By combining all the elements, it is clearly noticeable that all agilityrelated motor activity must be carried out on a specified basis. The terms planned and non-planned agility occur frequently in the literature which describes different agility movement structures. In pre-planned agility, recognized as a change-of direction speed (CODS), the order of appearing stimuli can be predicted by the participant. It means that the participants are aware of the exact movement pattern required before the start or have to react in response to given stimuli during the test, respectively. In regard to non-planned agility, also known as reactive agility (RA), the player is forced react to an emerging signal (sound, light or video-based player changing the direction) that is randomly selected by a person or system such as a computer. Therefore, in most situation in team sports when players are challenged to unknown match-play situations, agility tests that separate the cognitive skills from the motor elements of performance could be coun- terproductive when producing misleading interpretations [8].

In the theory and practice of sports there is considerable interest in the development of adequate diagnostic agility tests and specific training methods designed to improve agility. One of the many goals in this type of research is to create a technical basis and protocol for measuring pre-planned and non-planned agility. The authors have put forth a significant amount of effort into this task and have designed a battery of tests for assessing the differences between motor tasks of pre-planned (CODS) agility tasks and nonplanned (RA) agility tasks. The agility movements were executed in different directions: combined frontal, universal, semi-circular, and lateral planes. In this experiment we used universal agility components as the movement structures which appear most commonly in ball sports. Up until now the number of such studies has been relatively low, largely due to a lack of appropriate measurement technologies. However, the new FitLight Trainer technology enables participants to perform agility tests during the execution of pre-planned and non-planned movement structures, and allows for their direct comparison. Moreover, this technology is an efficient training tool for developing sport-specific agility performance.

The review article of Hojka et al. [21] focused on the analysis of change-of-direction movement, reactive agility, and any relationship between these skills and sprinting and jumping abilities. Same components have been proven to be independent of one another where some were proved to be related. Therefore, the analysis of the differences between the tests (pre-planned movement - CODS and non-planned (randomly selected) movement -RA) in the field of movement efficiency can let us specify the differences and independent movement characteristics of these physical skills which will allow for application of the right type of training and achieve the highest level of various agility types. In turn, such actions will allow for application of the right type of training in order to achieve the highest level of agility in regard to motor ability.

Therefore, the primary goal of the experiment was to examine the differences between reactive agility (RA) as non-planned (randomly selected stimuli) and changeof-direction speed (CODS) as pre-planned movement among various levels of sport performance - young soccer players and sport students. It was hypothesized that there would be comparable results between soccer players in the RA test and female student-athletes, although young soccer players have greater experience in using this kind of motor structure and better 
conditioning. The main difference in the level of fitness preparation (conditioning) between soccer players and students is that students demonstrate lack of experience in performing specific motion structures in the game (e.g. stops, change-of-direction, and restarts), which not only determines the effectiveness of the game but may also affect the performance level of the various forms of agility. It can be supposed that this may be due higher levels of anticipation, and the capacity for attention, which is limited with the amount of information provided to the brain.

\section{Material and methods}

\section{Sample of subjects}

Participants included 36 young soccer players (12 male and 24 female) and 58 physical education students (36 male and 22 female). The average age for soccer players was 19.1 ( \pm 1.18 ), and 20.9 years ( \pm 1.64) for physical education students. All of the soccer players (both male and female) were from various sports clubs. They participated in the university sports system and systems outside the school. Some athletes played in leagues, e.g. IV and V league, while others only played in student competitions like an inter-university league which university law allows. Team athletes were from a variety of clubs. The physical education male and female students were assigned do regular physicals activities, which resulted from their study program. All the subjects were active and fit and had no injury to their locomotor system at the time of measurements. The subjects were informed about the aim of the experiment and the measurement procedures and signed a written statement of consent in compliance with the Helsinki-Tokyo Declaration, which states that their consent to participate in the study is voluntary and can be withdrawn at any time. Parental consent was also obtained from those individuals under 18 years of age. The participants were instructed to maintain their normal intake of fluids and food during the experiment. Additionally, they were instructed to avoid any strenuous physical activity 24 hours prior to testing. All participants were familiarized with the test exercises at least one week before the beginning of the experiment.

\section{Test procedures}

The study used FitLight Trainer, a wireless system of interconnected light powered sensors manufactured by Sport Corp. Ontario, Canada. This system is used for measuring agility, reaction time, speed, and coordination. The system is comprised of seven LED powered lights and controlled by an Android application. Activation of each light module can be triggered by either direct contact or proximity. The system can be programmed as a sequence of light activations or deactivations of light rays by touch. Time is registered on the dashboard for each of the sensory units. The LEDs can be set for remoteness and sensitivity. In the study, the sensitivity was set to medium, with distance activation set to $20 \mathrm{~cm}$. The internal software package enables six random LED activation program sequences. The times recorded on the dashboard were manually entered into a MS Excel spread sheet.

The measurements were conducted within a time period of one week at the sports hall of the Faculty of Sport, under favourable climatic conditions. The flooring used was tartan, which enabled optimal performance of the experiment. After a 15-minute warm-up, the subjects were informed about the testing scenario. A demonstration of tests followed. Each test was performed twice and the better of the two results was then used for statistical analysis. The subjects were allowed a 3 to 5 minute break between repetitions. Reactive agility performance in all tests included movements which were completely unknown as the combination of tasks was randomly programmed by the computer.

Timing for the universal direction agility test (UA) began the moment each subject crossed the centrally located LED light signal with their hand, located in the center of the test course. When participants broke the base light signal, a computer ignited six LED lights placed on the top of 40-cm-high cones. A subject had to assess which LED light was flashing first, then react and run as fast as possible to that particular cone, cross a designated area of $20 \mathrm{~cm}$ around the light with their preferred hand, and return to the base light. After returning to the start line, participants were required to touch the top of the LED signal again, turn, and start running toward the next flashing LED light in the test course. Each time a subject crossed the center LED light the computer turned on one of the LED lights. The UA test trial consisted of one independently performed course, which was completed after subjects turned off the last light in the test and returning to the center LED light signal. Two trials of universal direction agility test (UA) were performed in the following designated scenarios (Figure 1):

1) In order to perform the first testing specification (pre-planned agility as the change of direction speed (CODS)) all subjects had knowledge of the order of 


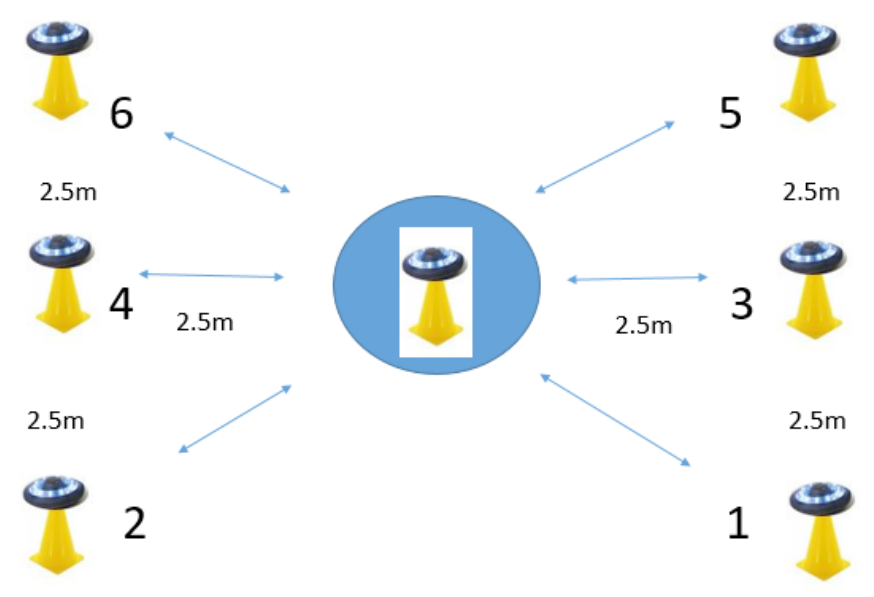

Figure 1. Universal direction agility test performed as Reactive Agility and Change of Direction speed

switching LED light in designated order: 1-2-3-4-5-6. The best result was retained as the final score.

2) In the second testing specification (non-planned agility as reactive agility) all subjects used the same tests scenario as in the CODS tests. However, the order of switching LED lights was randomly selected by the computer in a designated order: 6-3-2-4-1-5. The best result was retained as the final score.

\section{Statistical analysis}

The results obtained were then statistically processed using SPSS software package. For all tests, the basic descriptive statistical parameters were calculated in- cluding mean value, standard deviation, minimum, and maximum. The differences between the CODS and RA test scenarios were established by the result of the analysis of variance ANOVA ( $F$-value) at the level of characteristic features $p<0.01$ and $p<0.05$. The differences between male and female student athletes were also conducted.

\section{Ethical approval}

The research related to human use has been complied with all the relevant national regulations and institutional policies, has followed the tenets of the Declaration of Helsinki, and has been approved by the authors' institutional review board or an equivalent committee.

\section{Informed consent}

Informed consent has been obtained from all individuals included in this study.

\section{Results}

Table 1 shows anthropometric characteristics of the sub-sample. The average body height for soccer players was $181.8 \mathrm{~cm} \pm 5.6 \mathrm{~cm}$ for men, and 169.37 $\mathrm{cm} \pm 5.9 \mathrm{~cm}$ for women. In the students, both the male and female average body height was shorter by $1.25 \mathrm{~cm}$ and $2.8 \mathrm{~cm}$, respectively. Average body weight for male and female soccer players was $78.3 \mathrm{~kg} \pm 9.1 \mathrm{~kg}$ and $63.8 \mathrm{~kg} \pm 8.8 \mathrm{~kg}$, respectively. The students were slightly heavier than the athletes.

Table 1. Basic statistics (mean, standard deviation) of anthropometric characteristics.

The statistical significance was performed at the $p<0.05$

$\mathrm{N} \quad$ Mean $\quad$ St. deviation $\begin{gathered}95 \% \text { Confidence Interval } \\ \text { for Mean }\end{gathered} \quad \mathrm{F} \quad$ Sig.

\begin{tabular}{|c|c|c|c|c|c|c|c|c|}
\hline \multicolumn{9}{|l|}{ STUDENTS } \\
\hline \multirow{3}{*}{ Body height } & men & 36 & 180.55 & 6.1 & 178.7 & 183.9 & \multirow{3}{*}{65.208} & \multirow{3}{*}{$<0.001$} \\
\hline & women & 22 & 167.29 & 5.8 & 165.2 & 171.2 & & \\
\hline & total & 58 & 173.92 & 8.1 & 174.6 & 178.2 & & \\
\hline \multirow{3}{*}{ Body mass } & men & 36 & 79.1 & 8.8 & 74.8 & 83.1 & \multirow{3}{*}{33.935} & \multirow{3}{*}{$<0.001$} \\
\hline & women & 22 & 64.9 & 10.4 & 59.9 & 68.2 & & \\
\hline & total & 58 & 72.0 & 12.6 & 71.1 & 77.2 & & \\
\hline \multicolumn{9}{|c|}{ SOCCER PLAYERS } \\
\hline \multirow{3}{*}{ Body height } & \multirow{3}{*}{$\begin{array}{c}\text { men } \\
\text { women } \\
\text { total }\end{array}$} & 12 & 181.8 & 5.6 & 178.4 & 183.3 & \multirow{3}{*}{64.938} & \multirow{3}{*}{$<0.001$} \\
\hline & & 24 & 169.37 & 5.9 & 166.9 & 171.2 & & \\
\hline & & 36 & 175.58 & 8.3 & 173.9 & 178.2 & & \\
\hline \multirow{3}{*}{ Body mass } & men & 12 & 78.3 & 9.1 & 74.8 & 81.8 & \multirow{3}{*}{32.885} & \multirow{3}{*}{$<0.001$} \\
\hline & women & 24 & 63.8 & 8.8 & 59.2 & 67.5 & & \\
\hline & total & 36 & 71.45 & 11.2 & 69.1 & 76.9 & & \\
\hline
\end{tabular}




\section{HUMAN MOVEMENT}

S. Rauter et al., Reactive agility and change-of-direction speed

The statistics of the results (Table 2) show that soccer players were significant faster in selected CODS tests $(-1.73 \mathrm{~s} ; 13.1 \%)$ and RA tests $(-2.34 \mathrm{~s} ; 14.3 \%)$. The analysis of variance ANOVA indicated that the sub-samples (soccer players versus students) of subjects showed a statistically significant difference in the universal agility test scenario of CODS (pre-planned stimuli) $(p<0.001)$ and non-planned RA (randomly selected stimuli) $(p<0.001)$. Results also showed that male subjects, on average, did better than female subjects on pre-planned agility performance tasks (-0.47s; 5.5\%;). The greatest difference between male and female subjects was observed in the non- planned universal reactive agility UA-RA test $(-2.84 \mathrm{~s}$; $17.9 \%)$.

The results in Table 3 show gender comparison among soccer players and students was significant faster in UA-test of CODS $(-1.73 \mathrm{~s} ; 13.1 \%)$ and RA $(-2.34 \mathrm{~s} ; 14.3 \%)$. The analysis of variance ANOVA indicated statistically significant gender differences in the selected test of pre-planned (CODS) and nonplanned (RA) agility among soccer players and students. Results also showed (Table 3) that male students performed CODS better than female students $(-2.2 ; 16 \%)$. Among soccer player, there was a smaller difference between males and females but this dif-

Table 2. Basic statistics of reactive and non-reactive agility tests (mean, standard deviation), the analysis of variance of agility (F, sig.) between the sub-samples. The statistical significance was performed at $p<0.05$

\begin{tabular}{|c|c|c|c|c|c|c|c|c|c|}
\hline & & & & & & & & & \\
\hline & & & $\mathrm{N}$ & Mean & $\begin{array}{c}\text { St. } \\
\text { deviation }\end{array}$ & $\begin{array}{l}95 \% \mathrm{C} \\
\text { Interva }\end{array}$ & $\begin{array}{l}\text { idence } \\
\text { Mean }\end{array}$ & $\mathrm{F}$ & Sig. \\
\hline \multirow{3}{*}{ UA PP } & \multicolumn{2}{|c|}{ Soccer players } & 36 & 13.06 & 1.39 & 12.69 & 13.42 & & \\
\hline & \multicolumn{2}{|l|}{ Students } & 58 & 14.79 & 1.83 & 14.18 & 15.41 & 27.153 & $<0.001$ \\
\hline & \multicolumn{2}{|l|}{ Total } & 94 & 13.72 & 1.78 & 13.36 & 14,09 & & \\
\hline \multirow{3}{*}{ UA NP } & \multicolumn{2}{|c|}{ Soccer players } & 36 & 16.33 & 2.83 & 15.59 & 17.08 & & \\
\hline & \multicolumn{2}{|l|}{ Students } & 58 & 18.67 & 2.19 & 17.93 & 19.41 & 17.938 & $<0.001$ \\
\hline & \multicolumn{2}{|l|}{ Total } & 94 & 17.23 & 2.83 & 16.65 & 17.81 & & \\
\hline UA PP & & Men & 48 & 13.36 & 1.27 & 12.99 & 13.73 & & \\
\hline Women & & 46 & 14.10 & 2.14 & 13.47 & 14.74 & 4.213 & & .043 \\
\hline Total & & 94 & 13.72 & 1.78 & 13.36 & 14.09 & & & \\
\hline UA NP & & Men & 48 & 15.84 & 2.79 & 15.03 & 16.65 & & \\
\hline Women & & 46 & 18.68 & 2.04 & 18.08 & 19.29 & & 31.62 & $<0.001$ \\
\hline Total & & 94 & 17.23 & 2.83 & 16.65 & 17.81 & & & \\
\hline
\end{tabular}

UA-CODS - universal direction agility of CODS, UA-RA- universal direction agility of RA

Table 3. Basic statistics of reactive and non-reactive agility tests (mean, standard deviation) The analysis of variance of agility (F, sig.) between the sub-samples of students and soccer players.

The statistical significance was performed at $p<0.05$

\begin{tabular}{|c|c|c|c|c|c|}
\hline $\mathrm{N}$ & Mean & St. deviation & $\begin{array}{l}\text { 95\% Confidence Interval } \\
\text { for Mean }\end{array}$ & $\mathrm{F}$ & Sig. \\
\hline
\end{tabular}

\begin{tabular}{|c|c|c|c|c|c|c|c|c|}
\hline \multicolumn{9}{|c|}{ STUDENTS } \\
\hline \multirow[b]{2}{*}{ UA-CODS } & Men & 36 & 13.33 & 0.92 & 12.75 & 13.91 & \multirow{2}{*}{16.685} & \multirow{2}{*}{$<0.001$} \\
\hline & Women & 22 & 15.53 & 1.74 & 14.79 & 16.26 & & \\
\hline \multirow[b]{2}{*}{ UA-RA } & Men & 36 & 17.14 & 1.46 & 16.21 & 18.06 & \multirow{2}{*}{11.529} & \multirow{2}{*}{.002} \\
\hline & Women & 22 & 19.44 & 2.10 & 18.55 & 20.33 & & \\
\hline \multicolumn{9}{|c|}{ SOCCER PLAYERS } \\
\hline \multirow{3}{*}{ UA } & Men & 12 & 12.54 & 1.27 & 11.98 & 13.11 & \multirow{2}{*}{5.135} & \multirow{2}{*}{.027} \\
\hline & Women & 24 & 13.37 & 1.38 & 12.90 & 13.84 & & \\
\hline & Men & 12 & 15.40 & 3.00 & 14.39 & 16.42 & \multirow{2}{*}{12.355} & \multirow{2}{*}{.001} \\
\hline UA NP & Women & 24 & 17.86 & 1.65 & 17.12 & 18.59 & & \\
\hline
\end{tabular}

UA-CODS - universal direction agility of CODS, UA-RA- universal direction agility of RA 
ference was still significantly different (-0.83s; 6.6\%). The statistically significant difference between genders among soccer players and students was also found for the RA test.

\section{Discussion}

The development of state-of-the-art technologies has enabled the design of test scenarios and sites for the training of pre-planned CODS and non-planned (randomly selected stimuli) RA agility performance. The subjects performed CODS and RA tests in the same manner (testing scenario). First, they carried out the known movement routine, and then the unknown movement routine. The aim of study was also to identify any potential differences in the CODS and RA tests between the sub-samples of young soccer players and the group of sport students, including gender-related differences.

Movement, which is not pre-defined, evidently requires more controlled biomotor, cognitive, and sensory skills than movements where the pattern is known in advance. Non-planned, or reactive agility depends on effective anticipation, visual perceptive speed, reaction speed, and the capacity for attention which is limited by the quantity of information and the quantity of the active working memory [8]. The decisive factors in the selected tests are a high concentration level, 360-degree visual scanning, scope of attention, and reaction time. The execution of movement is highly complex, movement control is limited, and the subject is required to operate in a multi-dimensional space. Sensors are activated outside the field of vision in the forward, sideways, and backward directions.

The results of the study indicate statistically significant differences in a universal direction agility test of pre-planned (CODS) and non-planned (RA) between soccer players and physical education students. In general, soccer players were significantly faster in selected tests of pre-planned and non-planned agility. The statistically significant differences in the CODS and RA tests between men and women were hypothetically generated by the higher natural motor potential of male subjects, in particular regarding explosive power, reactive eccentric/concentric strength, acceleration speed, and deceleration speed. In terms of "agility motor skills", men dominate over women. The differences between the pre-planned and nonplanned agility tests by female subjects give us slightly different pictures in comparison to male subjects. There is a considerable gap between the reactive and non-reactive agility performance found in test, which amounts to more than $30 \%$.
In summary, the results of the study showed that agility, as a motor ability, is a complex issue which depends on many psychological and movement factors and the interaction among them. These kinds of preplanned (CODS) and non-planned (RA) agility tests were found to be a useful diagnostic tool for assessing different types of agility movement structures. The limitations in this study were the analysis of only one test with a medium level of complexity. The use of more complex agility tests would make for interesting results and comparisons. Additionally, the hypothesis that the results of soccer players in the RA test would be comparable among female student athletes was not fully confirmed. The greater conditioning level of young soccer players showed superiority over some cognitive factors. It can be expected that with a more comprehensive (complex) test the results would have been different and challenge this hypothesis.

\section{Conclusions}

Soccer players were significant faster in selected test of CODS and RA. The greatest difference between male and female subjects was observed in the nonplanned universal direction reactive agility test, UA-RA. The analysis of variance ANOVA indicated statistically significant gender differences in the selected test of preplanned (non-reactive) and non-planned (reactive) agility among soccer players and students. The differentiation of the pre-planned and non-planned agility tests pointed to different bio motor abilities which evidently define the performance of athletes. The practical message for coaches and related professionals which results from this experiment is that agility in team sports (including soccer) as a motor skill requires specific physical qualities and skills. Therefore, agility should be evaluated and diagnosed via separate assessments. The more important message is that agility must be developed through a different training regime, which must include sport-specific (activities) and take gender into account. This mainly applies to decisionmaking movement structures (RA) that are often more challenging during sport-specific performance. These movements are not much different from motor ability potential or stronger morphological components.

\section{Acknowledgements}

The study was the product of cooperation between the Faculty of Sport, Institute of Sport, University of Ljubljana, and University School of Physical Education in Wroclaw, Poland. 


\section{Disclosure statement}

No author has any financial interest or received any financial benefit from this research.

\section{Conflict of interest}

The authors state no conflict of interest.

\section{References}

1. Bidaurrazaga-Letona I, Carvalho HM, Lekue JA, Badiola A, Figueiredo AJ, Gil SM. Applicability of an agility test in young players in the soccer field. Rev Bras Med Esporte. 2015;21(2):133-138; doi: 10.1590/1517869220152102144406.

2. Malina RM, Ribeiro B, Aroso J, Cumming SP. Characteristics of youth soccer players aged 13-15 years. Br J Sports Med. 2007;41(5):290-295; doi: 10.1136/bjsm. 2006.031294.

3. Larkin P, O’Connor D. Talent identification and recruitment in youth soccer: Recruiter's perceptions of the key attributes for player recruitment. Plos One. 2017; 12(4). doi: 10.1371/journal.pone.0175716.

4. Bidaurrazaga-Letona I, Carvalho HM, Lekue JA, Badiola A, Santos-Concejero J, Gil SM. Longitudinal field test assessment in a Basque soccer youth academy: a multilevel modeling framework to partition effects of maturation. Int J Sports Med. 2015;36(3):234-240; doi: 10.1055/s-0034-1385881.

5. Bangsbo J, Iaia FM, Krustrup P. Metabolic response and fatigue in soccer. Int J Sports Physiol Perform. 2007; 2(2):111-127. doi: 10.1123/ijspp.2.2.111.

6. Vescovi JD, Rupf R, Brown TD, Marques MC. Physical performance characteristics of high-level female soccer players 12-21 years of age. Scand J Med Sci Sports. 2011;21(5):670-678.doi:10.1111/j.1600-0838.2009.01081.

7. Lockie RG, Jeffriess MD, McGann TS, Callaghan SJ, Schultz AB. Planned and reactive agility performance in semi-professional and amateur basketball players. Int J Sports Physiol Perform. 2014;9(5):766-771; doi: 10.1123/ijspp.2013-0324.

8. Benvenuti C, Minganti C, Condello G, Capranica L, Tessitore A. Agility assessment in female futsal and soccer players. Medicina (Kaunas). 2010;46(6):415-420.

9. Sheppard JM, Young WB. Agility Literature Review: Classifications, Training and Testing. J Sports Sci. 2006; 24(9):919-932; doi: 10.1080/02640410500457109.

10. Young WB, Willey B. Analysis of a reactive agility field test. J Sci Med Sport. 2010;13(3):376-378; doi: 10.1016/j.jsams.2009.05.006

11. Hodges N, Starks J. MacMahon C. Expert Performance in Sport: A Cognitive Perspective. In: Ericsson KA, Charness N, Fletovich PJ, editors. The Cambridge Handbook of Expertise And Expert Performance. Cambridge: University Press; 2006; p. 71-488.

12. Little T, Williams AG. Specificity of acceleration, maximum speed, and agility in professional soccer players. J Strength Cond Res. 2005;19(1):76-78. doi: 10.1519/ 14253.1.
13. Spasic M, Krolo A, Zenic N, Delextrat A, Sekulic D. Reactive Agility Performance in Handball; Development and Evaluation of a Sport-Specific Measurement Protocol. J Sports Sci Med. 2015;14(3):501-506.

14. Laessoe U, Grarup B, Bangshaab J. The Use of Cognitive Cues for Anticipatory Strategies in a Dynamic Postural Control Task - Validation of a Novel Approach to Dual-Task Testing. Plos One. 2016;11(8); doi:10.1371/ journal.phone.0157421.

15. Enoka R. Neuromechanics of Human Movement. 3rd ed. Champaign: Human Kinetics; 2003.

16. Gabbett TJ, Kelly JN, Sheppard JM. Speed, Change of direction speed, and reactive agility of rugby league players. J Strength Cond Res. 2008;22(1):174-181; doi: 10.1519/JSC.0b013e31815ef700.

17. Reilly T, Williams AM, Nevill A, Franks A. A multidisciplinary approach to talent identification in soccer. J Sports Sci. 2000;18(9):695-702; doi: 10.1080/0264041 0050120078.

18. Svensson M, Drust B. Testing soccer players. J Sports Sci.2005;23(6):601-618; doi: 10.1080/026404104000 21294.

19. Figueiredo AJ, Gonçalves CE, Coelho E Silva MJ, Malina RM. Characteristics of youth soccer players who drop out, persist or move up. J Sports Sci. 2009;27(9):883891; doi: 10.1080/02640410902946469.

20. Gil S, Ruiz F, Irazusta A, Gil J, Irazusta J. Selection of young soccer players in terms of anthropometric and physiological factors. J Sports Med Phys Fitness. 2007; 47(1):25-32.

21. Hojka V, Stastny P, Rehak T, Gołas A, Mostowik A, Zawart M, Musálek M. A systematic review of the main factors that determine agility in sport using structural equation modeling. J Hum Kinet. 2016;52(1):115123; doi: 10.1515/hukin-2015-0199. 